\title{
A virtual reality study in university classrooms: The influence of classroom colour on memory and attention.
}

\author{
Juan Luis Higuera-Trujillo ${ }^{\text {ac}}$, Carmen Llinares Millán ${ }^{\text {ac }}$, Susana Iñarra Abad ${ }^{\text {bc }}$, Juan \\ Serra Lluch ${ }^{\text {b }}$ \\ ${ }^{a}$ Universitat Politècnica de València-ETSIE, ${ }^{b}$ Universitat Politècnica de València-ETSA, \\ ${ }^{\mathrm{c}}$ Universitat Politècnica de València-I3B
}

\begin{abstract}
Design of teaching spaces influences the cognitive abilities of its users. Among the design variables, the colour stands out for the ease of its implementation and its aesthetic possibilities. Previous studies suggest that it can influence students' academic progress. However, due to the difficulty in studying their combinations, only a limited number of colours have been exhaustively studied. This was the objective of the present study: to contribute to the study of the effect of different colour parameters applied on the walls of university classrooms on students' memory and attention performances. To address it, a virtual reality study was carried out with 80 university students. The colour variable was studied through two parameters: hue (8 settings) and saturation (2 settings). The resulting 16 combinations were implemented in a virtual reality university classroom. Memory performance was quantified using a psychological task of remembering an auditory word list, and attention was quantified by the reaction time to auditory stimuli. Analyses indicate that memory and attention performance is affected by some of these parameters, so they could be especially critical in the design of this type of space. Results may be of interest to different agents involved in the university classroom project, from architects and designers to the political leaders of these institutions.
\end{abstract}

Keywords: Virtual reality, university classrooms, colour, memory, attention. 


\section{Introduction}

Colour is a powerful design element for architectural environments that has an impact in psychological and physiological behaviour. Previous studies have shown that wall colours in classrooms can have a significant influence in students' learning performance and emotions, and in consequence of this, in their academic achievement (Al-Ayash et al., 2015; Gaines \& Curry, 2011).

In Barret's work (2017) the impact of the physical design of classrooms during the learning progress has been summarize by three factors: Level of stimulation, Individualization and Naturalness. Each design parameter (light, sound, temperature, colour...) belongs to one of this factors. Thus, colour has un significant impact in factor "Level of stimulation", that, in turn, can explain 23\% the Overall Progress (Reading, Writing and Maths) in primary aged school children. (Barrett et al., 2015).

In a perceptive level colour can be described by 3 perceptual variables: hue, Value and Saturation. Hue corresponds to the dominant wavelength of the physical stimulus, the value refers to the lightness or darkness of a colour, while saturation (or chroma) is the intensity of the perceived color and ranges from gray (dull) to the maximum purity for a hue (bright).

Concerning the studies carried out so far, we can highlight three aspects:

1. Value is the variable which has been more analysed, however, several studies have shown that saturation has more influence than hue in human's emotional responses (Gao \& Xin, 2006) .

2. Although many research studies shows that colour has an influence on cognitive performances, most of them refers to mental attention (Elif et al., 2012). But here are others cognitive functions, such us memory, that seem to be crucial in learning process.

3. Most of the studies carried out in real physical spaces (Al ayash, 2015; Yildirim et al., 2015). The use of real stimuli presents two drawbacks, on the one hand, the high cost of modifying the real space and, on the other hand, the difficulty of limiting the set of environmental variables, particularly natural light. Thus, in this study we use immersive virtual environments (IVE) able to create simulated space under controlled conditions. These immersive systems provide the advantage of simulating the environment in conditions perfectly controlled, at low cost. They also allow the cognitive response to be recorded with better predictive information regarding performance in a real environment (Rizzo et al., 2000).

The aim of this paper is to analyse the impact that hue and colour saturation of classroom walls have on attention and memory performances in university students. 


\section{Materials and Methods}

The experimental approach consisted of a laboratory fieldwork. The participants (Figure 1) performed psychological tasks focused on memory and attention performance during immersion in virtual classrooms.

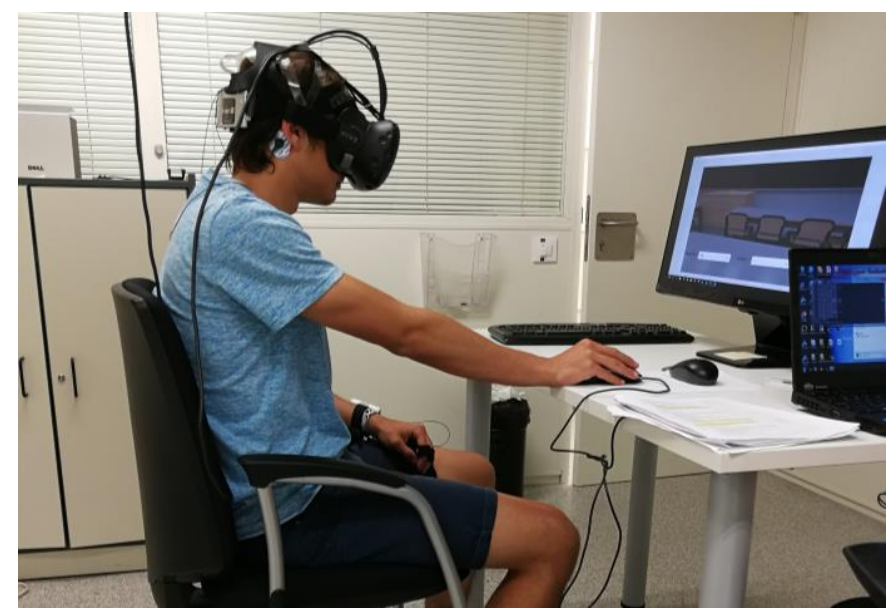

Fig. 1: Participant during the psychological tasks.

Source: own elaboration.

\subsection{Participants}

80 subjects participated in the study. The sample was gender-balanced (40 women and 40 men) and the average age was $23.21(\sigma=3.156)$. Regarding the inclusion criteria, three aspects were established: (1) being a university student, (2) being Spanish (to avoid possible cultural effects), and (3) having normal or corrected-to-normal vision with contact lenses without colour deficiencies.

\subsection{Classroom}

A real physical classroom was used as a base. Specifically, the base classroom was one from the Higher Technical School of Building Engineering (ETSIE) of the Universitat Politècnica de València. Its virtual replica was made, and different configurations of wall colour were implemented. Two parameters were studied: (1) "hue"; and (2) "saturation" (or chroma). The configurations of each one were: for hue (1a) "5BG", (1b) "5PB", (1c) "5P"; (1d) "5RP"; (1e) "5R"; (1f) "5YR"; (1g) "5Y"; (1h) "5GY"; and for saturation (2a) "high saturation, 10-16", and (2b) "low saturation, 4-10". Their combination resulted in 16 simulations (Figure 2). All of them were rendered in $360^{\circ}$ format and showed using the head-mounted device "HTC Vive". 


\subsection{Psychological records}

The psychological tasks were focused on quantify the memory and the attention performance.

\subsubsection{Memory task}

The psychological memory task was similar to the DRM paradigm tests (Beato \& Díez 2011). The task consisted on remembering the 15 words of three pre-recorded lists (45 total words). The participant had to repeat the words of each list in a 30 seconds a time limit. The next list was then listened, until the task was finished. The number of words remembered was taken as a quantification of memory.

\subsubsection{Attention task}

The psychological attention task was similar to the continuous auditory performance tests (Seidman et al. 1998). The task consisted on reacting as soon as possible to specific auditory stimuli (24 objectives) and to avoid others (96 distractors). All these stimuli were shown randomly, with a minimum of $800 \mathrm{~ms}$ and a maximum of $1600 \mathrm{~ms}$. The reaction time was taken as the attention quantification.

\subsection{Data processing}

The database was collected and anonymized, and the statistical analysis were carried out using IBM SPSS v.16.0 software. 
Juan Luis Higuera-Trujillo, Carmen Llinares Millán, Juan Serra Lluch, Susana Iñarra Abad

SIMULATION
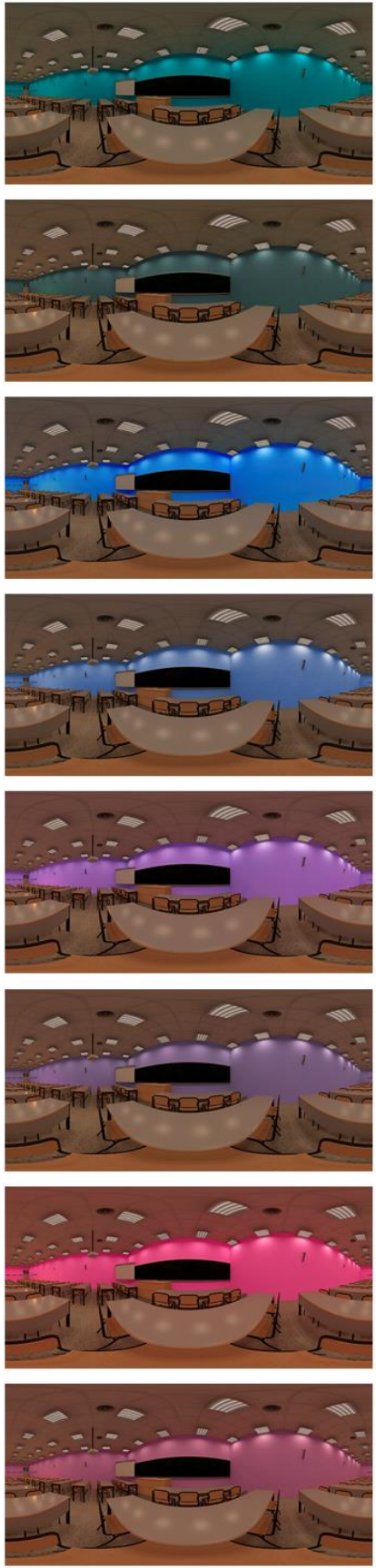

HUE CHR
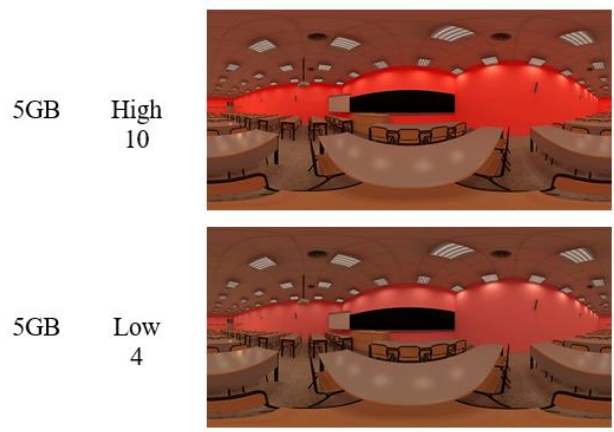

$5 \mathrm{~PB}$

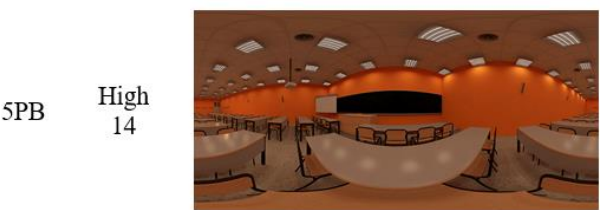

5PB

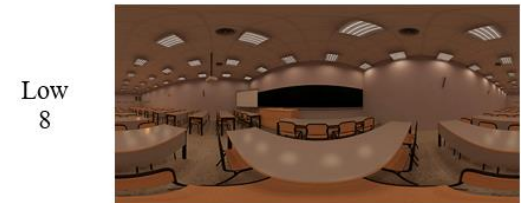

$5 \mathrm{P}$

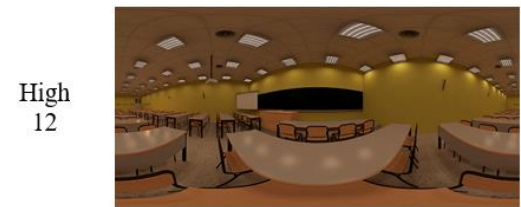

$5 \mathrm{P}$

$5 R P$

$5 \mathrm{RP}$
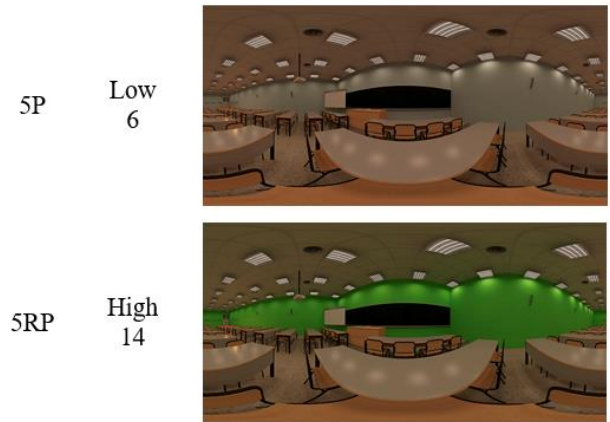

Low
8
HUE CHR

5R $\quad \begin{gathered}\text { High } \\ 16\end{gathered}$

$\begin{array}{cc} & \text { Low } \\ 5 \mathrm{R} & 10\end{array}$

5YR High

5YR 4

$5 Y \quad$ High

$5 Y$

Low

5GY High

$5 \mathrm{GY}$

Low

Fig. 2: Wall colour simulations.

Font: own elaboration.

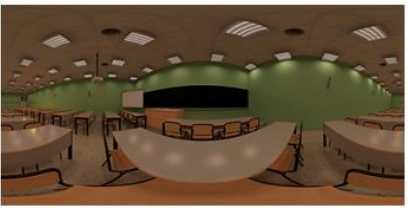

4 


\section{Results}

Results are organized in the following sections: (3.1) distribution of the variables; and (3.2) influence of wall colour on memory and attention performance.

\subsection{Variable distribution}

First, the distribution of the variables was checked. The Kolmogorov-Smirnov test shows that memory $(p=0.612)$ and attention $(p=0.138)$ followed a normal distribution. The differences for each of the design variables are analysed using ANOVA test.

\subsection{Influence of geometry on memory and attention performance}

Analyses carried out allowed quantifying the effect of wall colour on attention and memory. Figure 3 shows the results obtained. The results are presented below, differentiating each of the two parameters: hue and saturation.

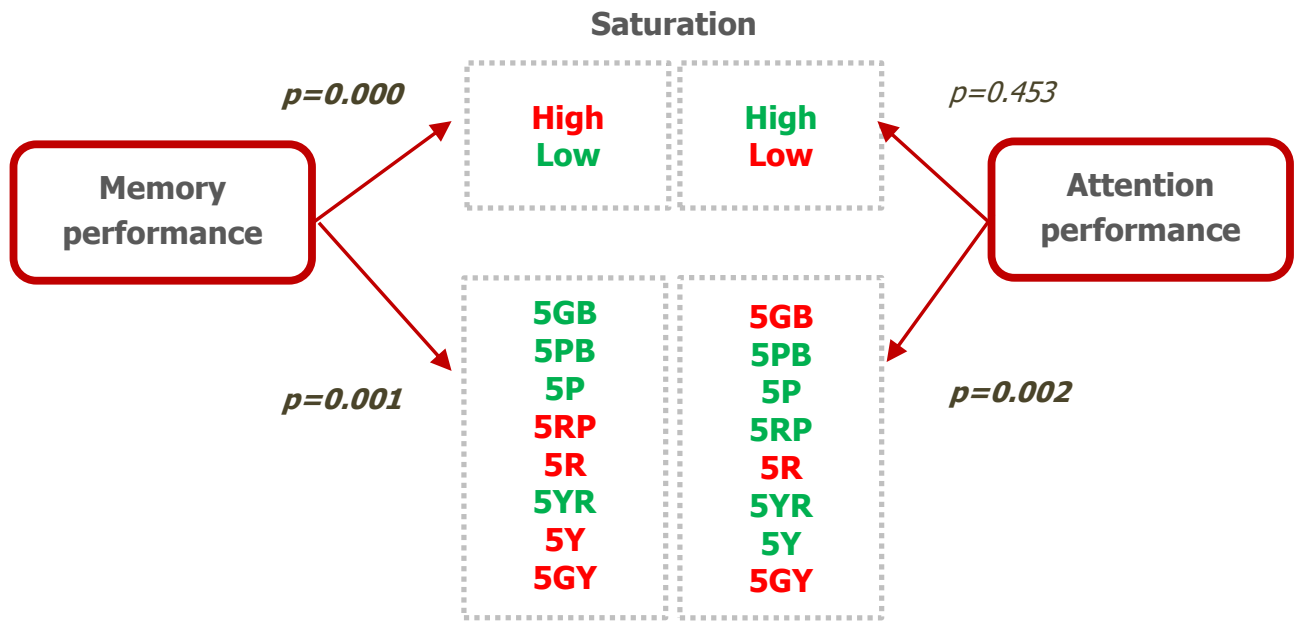

Hue

Fig. 3: Significant differences in memory and attention tasks.

Green and red highlight the best and worst results, respectively.

Font: own elaboration.

\subsubsection{Huе}

The analyses suggest that hue have a significant impact on both memory $(p=0.001)$ and attention $(\mathrm{p}=0.002)$ performances. Regarding memory performance, the hues that generate the best results are 5GB, 5PB, 5P and 5YR. Regarding attention performance, the hues are 5PB, 5P, 5RP, 5YR, and 5Y. 


\subsubsection{Saturation}

Saturation seems to have a significant impact on memory $(\mathrm{p}=0.000)$. Wall colours with low saturation contribute to students' improved memory performance. However, saturation does not have a significant impact on attention performance $(\mathrm{p}=0.453)$.

\section{Conclusions}

Analyses indicate that wall colour may affects both the memory and attention performances. The guidelines match in some of the hues: 5PB, 5P, and 5YR. Although confirmatory studies are needed, the results seem to indicate that purple or blue-purple (cold hues) contributes to enhance the cognitive performance in memory and attention. Regarding saturation, the guidelines could be opposed to improve attention and memory. However, since no significant differences in attention performance have been found, as a general guideline, it could be established that low saturations are better if the goal is to improve these cognitive functions. Considering that colour is a relatively easy design variable to implement in physical classrooms, these results may be useful for both new and existing classrooms.

\section{Acknowledgments}

This work was supported by the Ministerio de Economía, Industria y Competitividad of Spain (Project BIA2017-86157-R, and PRE2018-084051)

\section{References}

Al-Ayash, A., Kane, R. T., Smith, D., \& Green-Armytage, P. (2015). The influence of color on student emotion, heart rate, and performance in learning environments. Color Research and Application, 41(2). 196-205.

Barrett, P., Davies, F., Zhang, Y., \& Barrett, L. (2015). The impact of classroom design on pupils' learning: Final results of a holistic, multi-level analysis. Building and Environment, 89, 118-133.

Barrett, P., Davies, F., Zhang, Y., \& Barrett, L. (2017). The Holistic Impact of Classroom Spaces on Learning in Specific Subjects. Environment and Behavior, 49(4), 425-451.

Beato, M. S., \& Díez, E. (2011). False Recognition Production Indexes in Spanish for 60 DRM Lists with Three Critical Words. Behavior Research Methods 43 (2), 499-507. 
Elif, Yilmazer, S., \& Ural, S. E. (2012). The effects of achromatic and chromatic color schemes on participants' task performance in and appraisals of an office environment. Color Research and Application, 37(5), 359-366.

Gaines, K. S., \& Curry, Z. D. (2011). The Inclusive Classroom: The Effects of Color on Learning and Behavior. Journal of Family \& Consumer Sciences Education, 29(1), 46-57.

Gao, X. P., \& Xin, J. H. (2006). Investigation of human's emotional responses on colors. Color Research and Application, 31(5), 411-417.

Rizzo, A. A., Buckwalter, J. G., Bowerly, T., Van Der Zaag, C., Humphrey, L., Neumann, U. \& Sisemore, D. (2000). The virtual classroom: a virtual reality environment for the assessment and rehabilitation of attention deficits. CyberPsychology \& Behavior, 3(3), 483-499.

Seidman, L. J., Breiter, H. C., Goodman, J. M, Goldstein, J.M., Woodruff, P. W., O'Craven, K., Rosen, B. R.,Tsuang, M. T. \& Rosen, B. R. (1998). A Functional Magnetic Resonance Imaging Study of Auditory Vigilance with Low and High Information Processing Demand. Neuropsychology 12 (4), 505-18.

Yildirim, K., Cagatay, K., \& Ayalp, N. (2015). Effect of wall colour on the perception of classrooms. Indoor and Built Environment, 24(5), 607-616. 